\title{
Industrialización y competitividad industrial en el mundo
}

Recepción: Mayo de 2006 / Aceptación: Junio de 2006

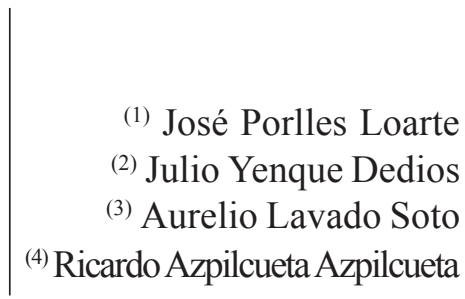

\section{RESUMEN}

El proceso de industrialización mundial poneen evidencia que América Latina y EI Caribe (ALC) no cuenta con capacidad competitiva industrial para hacer frente a otras regiones del mundo en desarrollo, como es el caso de los países de Asia del Este, perdiendo oportunidades para incrementar su presencia en las actuales tendencias industriales. El caso del Perú es preocupante, puesto que es uno de los 40 países peor clasificados por la ONUDI en posición competitiva industrial. Con el propósito de realizar una radiografía del mapa de la actividad industrial mundial y el desempeño industrial, se ha considerado entre otros documentos losúltimos Informes de Desarrollo Industrial de la ONUDI.

Palabras Clave: Tendencias industriales, rendimiento, manufacturas, tecnología, posicionamiento de mercado.

INDUSTRIALIZATION AND INDUSTRIAL COMPETITIVENESS IN THE WORLD ABSTRACT

The process of world industrialization puts in evidence that Latin America and The Caribbean (ALC) does not count with industrial competitive capacity to face other regions of the world in development, as it is the case of the countries of Eastern Asia, losing opportunities to increase its presence in the current industrial tendencies. The case of Peru is worrying, since it is one of the worst 40 countries classified for the UNIDO in industrial competitive position. With the object of carrying out an $x-$ ray of the map of the industrial activity in the world and the industrial performance, there have been considered some documents, among others, the last Reports of Industrial Development of the UNIDO.

Key words: Industrial tendencies, performance, manufactures, technology, positioning of market.

\section{INTRODUCCIÓN}

La erradicación de la pobreza como objetivo central del desarrollo mundial sólo se alcanzará con un indispensable crecimiento económico (Figura 1); así lo anota el Banco Mundial (2) y la ONUDI (9).

EI PNUD (13) revela que los indicadores sociales mejoran a un ritmo demasiado lento y ALC presenta la peor distribución de ingresos del mundo.

La ONUDI (6) afirma “...que el desarrollo productivo es la fuerza motriz de la aplicación de nuevas tecnologías a la producción y la fuente y el agente más importante de la innovación tecnológica; crea nuevas aptitudes y actitudes ante el trabajo, cataliza el cambio institucional y genera capacidades empresariales modernas". En este sentido a la industrialización le corresponde el papel crítico de ayudar a potenciar el crecimiento económico y social.

Un proceso de industrialización sostenido implica continua elevación de la productividad, lo cual es equivalente a progreso tecnológico. Como se apreciará más adelante, el fortalecimiento del sector industrial es la fuente de un sostenido incremento del bienestar de la sociedad, convirtiéndose en la herramienta de la reducción sostenida de la pobreza. Esta convicción del efecto directo e indirecto de la industria en su contribución al producto total, ha motivado el presente trabajo que se propone los objetivos siguientes:

1. Visualizar el desempeño industrial mundial.

2. Identificar factores claves que impulsan el desarrollo industrial.

3. Comprender el papel de la tecnología.

4. Tomar conciencia de la necesidad de un cambio del modelo industrial del Perú.

El papel de la Industria en el desarrollo general y la reducción de la pobreza muestra, a nivel mundial, que la mayor parte de las economías dotadas de recursos naturales no han tenido mucho éxito en los intentos de convertir los ingresos en crecimiento sostenible. Es así que, varios países del Africa y ALC, incluyendo el Perú han desperdiciado claramente la oportunidad de transformar ingresos procedentes de los recursos naturales en crecimiento sostenible. En el caso especial de algunos de los países no cabe la menor duda de que la incapacidad de industrializarse no ha sido

(1) Magíster en Administración. Profesor del Departamento de Análisis y Diseño de Procesos, UNMSM E-mail: joseporlles@yahoo.com

(2) Ingeniero Industrial. Profesor del Departamento de Diseño y Tecnología Industrial, UNMSM. E-mail: jyenque@yahoo.es

(3) Ingeniero Químico. Profesor del Departamento de Diseño y Tecnología Industrial, UNMSM. E-mail:mlavados@unmsm.edu.pe

(4) Ingeniero Industrial. Profesor de la Facultad de Ciencias Administrativas, UNMSM. E-mail: raasolimana@hotmail.com 


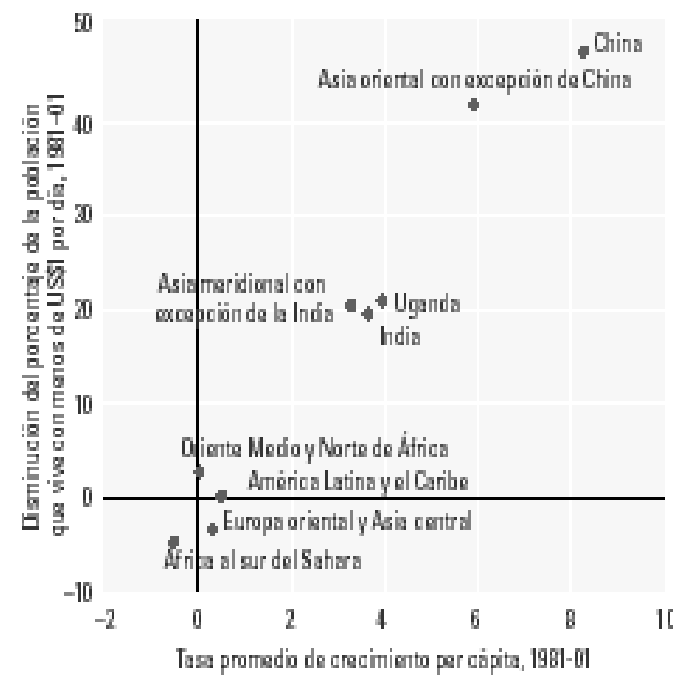

Figura 1. La reducción de la pobreza está estrechamente vinculada al crecimiento

Fuente: Banco Mundial, 2005

consecuencia de la falta de capital; así lo evidencia el caso de los países denominados "Tigres del Asia".

Al respecto, la ONUDI (7) considera más importante crear capacidades; hacer funcionar las fábricas a niveles competitivos, aumentar la calidad, introducir productos nuevos, modernizar las prácticas y diversificarse en actividades con mayor valor agregado. Si bien para ello también se requiere inversiones, al margen del clima adecuado para precipitarlas, se necesita un conjunto de recursos más valiosos que el dinero: aptitudes, organización, conocimientos, información, tecnología e instituciones: hacer mejor las cosas.

En la Figura 2 se ilustran los factores determinantes para la competitividad industrial, y en la Figura 3 se visualiza la ubicación de los países y sobretodo las regiones más dinámicas en el desarrollo industrial.

\section{MEDICION DEL RENDIMIENTO INDUSTRIAL COMPETITIVO}

La ONUDI (4) ha construido una metodología para medir el rendimiento industrial a través del Indice de Desempeño Industrial o Competitive Industrial Perfomance (CIP), basado en los siguientes indicadores centrales:

a. El valor agregado manufacturero (MVA). EI MVA per cápita define el nivel promedio del ingreso industrial, como una medida similar al producto per cápita de una economía.

b. Las exportaciones de manufactura (Mfd). El Mfd per cápita como una medida de la intensidad de las exportaciones manufactureras.

La Figura 4 permite apreciar el tablero de indicadores industriales y clasifica a los países de acuerdo a su rendimiento industrial competitivo y los factores determinantes de la competitividad industrial. Analiza econométricamente el impacto que cada factor ha tenido en la competitividad industrial; asimismo iden-

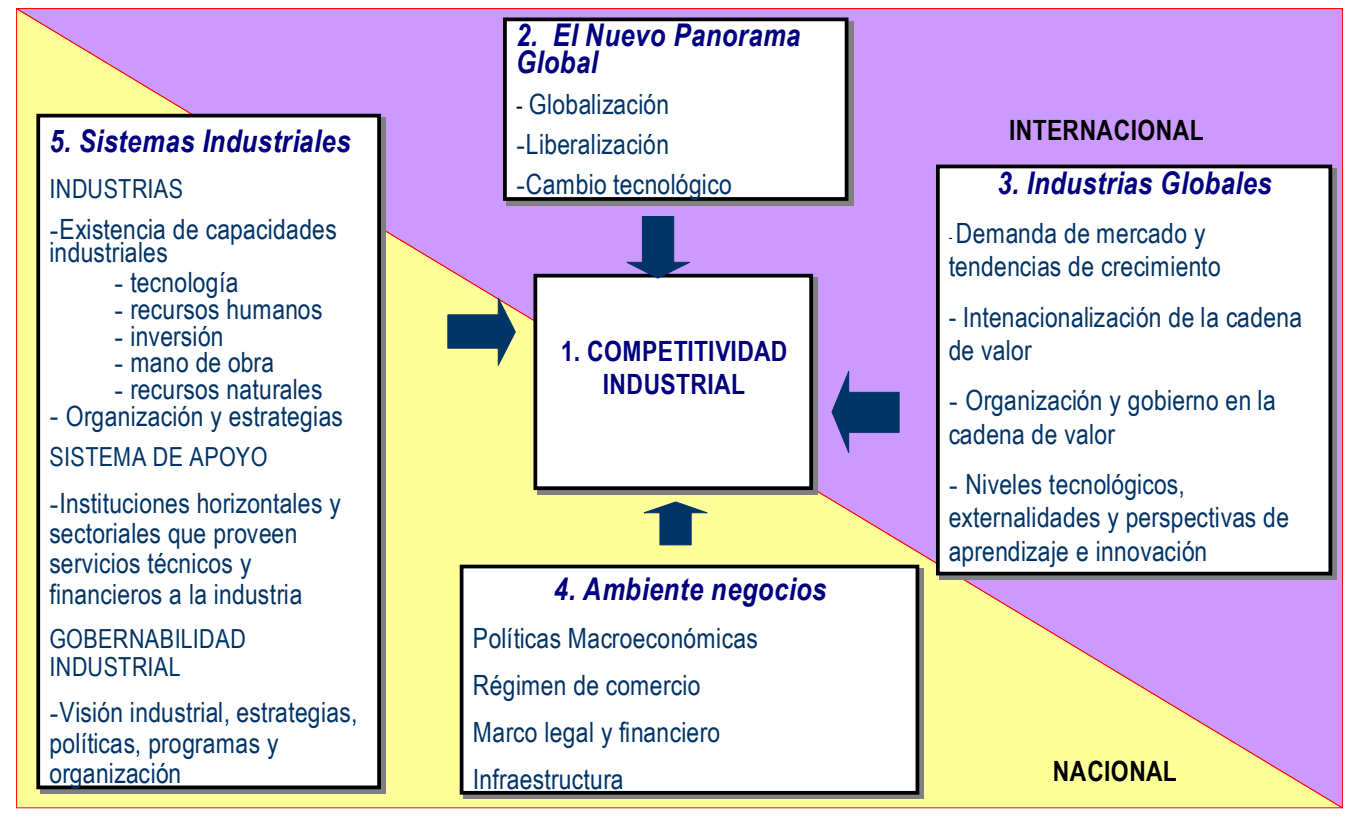

Figura 2. Factores determinantes para la Competitividad Industrial Fuente: ONUDI, 2004 


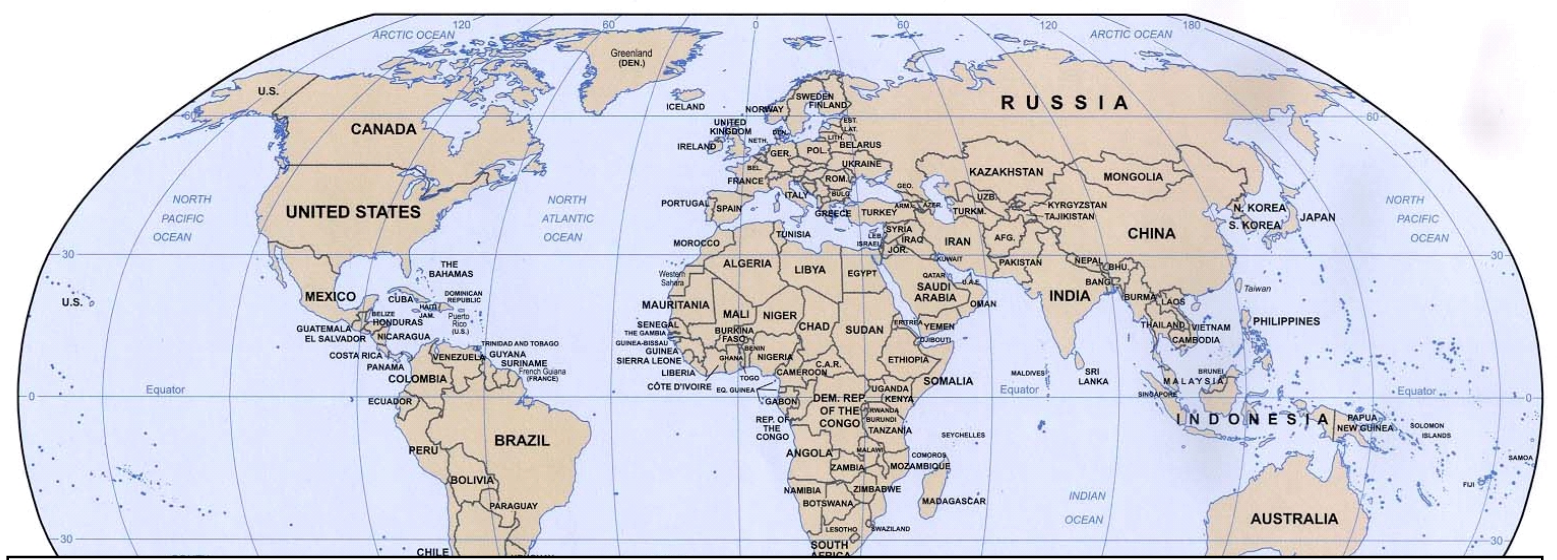

Países Industriales: USA, Japón, países europeos maduros...,

Economías en Desarrollo: ALC, Tigres del Asia (Singapur, Corea del Sur, Malasia, Taiwán, Tailandia....), China, algunos países de Sud Africa (SA) como Africa del Sur y ASS.

Economías en Transición: Países de la ex URSS, Rusia, Hungría, Polonia....

Países con desarrollo Mínimo (LDCs): algunos de ASS, Yemen, Nepal, Haití, Bangladesh...

Asia del Sur: India, Pakistán, Bangladesh...; Oriente Medio: Turkía, Egipto, Kuwait, Arabia Saudita, Omán.....

Asia del Este: China, Singapur, Corea, Malasia, Tailandia...; ASS: Africa subsahariana

Figura 3 . Visualización mundial (ubicación de regiones y países)

Fuente: www.viajeros.com, Enero 2002

tifica las diferentes estrategias que han seguido los países exitosos.

A diferencia de otros indicadores de competitividad, como el realizado por el World Economic Forum (WEF), el CIP involucra el ingreso per cápita industrial y las relaciones entre factores determinantes y la competitividad sólo al ámbito industrial, lo que facilita la identificación y cuantificación de variables input y output y sus vinculaciones. Permite visualizar los factores claves que han favorecido o no el desarrollo industrial de los países, distinguiendo entre variables del medio ambiente-económico y las variables estructurales claves para la competitividad industrial.

Cabe indicar que la ONUDI ha realizado innumerables aplicaciones de esta metodología en diferentes países del mundo, para que los gobiernos estén en mejor capacidad de establecer políticas sectoriales de desarrollo industrial.

\section{RANKING DE COMPETITITIVIDAD INDUSTRIAL MUNDIAL}

¿Cómo ha sido el desempeño industrial en el mundo y cómo lo mide la oNUDI?

El estudio comprende la evolución en el periodo 1980 al 2000, como se aprecia en el Cuadro 1. Singapur (considerado país en desarrollo) registra el mejor desempeño mundial en los últimos 20 años. Irlanda es la novedad y salta al segundo puesto en el 2000, luego de haber ocupado el puesto 19 en 1980. Es interesante anotar que ambos países siguieron estrategias similares ingresando en cadenas mundiales de alta tecnología y desarrollando un buen capital humano y una firme infraestructura.

En la Unión Europea (UE), los países industriales maduros han deteriorado su posición industrial, salvo Finlandia, que es el país más competitivo del mundo según el Indice de Competitividad para el Crecimiento de la WEF(11). Japón muestra una lenta pérdida de posiciones y USA también una ligera recupera-

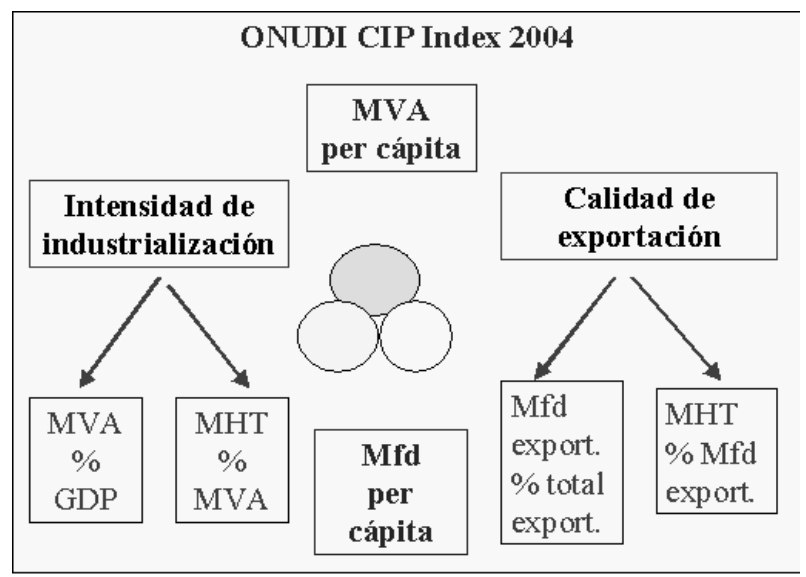

Figura 4. Indicadores de desempeño industrial Fuente: ONUDI CIP, 2004 
Cuadro 1. Ranking índice de desempeño industrial

\begin{tabular}{|l|r|r|r|}
\hline Economía & 2000 & 1990 & 1980 \\
\hline Singapur & 1 & 1 & 2 \\
\hline Irlanda & 2 & 9 & 19 \\
\hline Suiza & 3 & 2 & 1 \\
\hline Finlandia & 4 & 7 & 9 \\
\hline Suecia & 5 & 5 & 4 \\
\hline Japón & 6 & 4 & 5 \\
\hline Alemania & 7 & 3 & 3 \\
\hline USA & 11 & 14 & 13 \\
\hline Malasia & 15 & 23 & 40 \\
\hline Italia & 16 & 11 & 10 \\
\hline UK & 17 & 13 & 12 \\
\hline Tailandia & 23 & 32 & 47 \\
\hline China & 24 & 26 & 39 \\
\hline México & 26 & 29 & 31 \\
\hline Brasil & 31 & 27 & 24 \\
\hline India & 40 & 36 & 38 \\
\hline Colombia & 61 & 58 & 58 \\
\hline Chile & 64 & 62 & 50 \\
\hline Perú & 65 & 68 & 41 \\
\hline Venezuela & 66 & 60 & 68 \\
\hline Ecuador & 76 & 81 & 67 \\
\hline Total países & 93 & 93 & 93 \\
\hline
\end{tabular}

Fuente:ONUDI; Informe sobre el Desarrollo Industrial 2004, publicado el 20.07.04, pag 13-14. (El Informe abarca 155 países, pero el cuadro sólo representa una muestra básica).

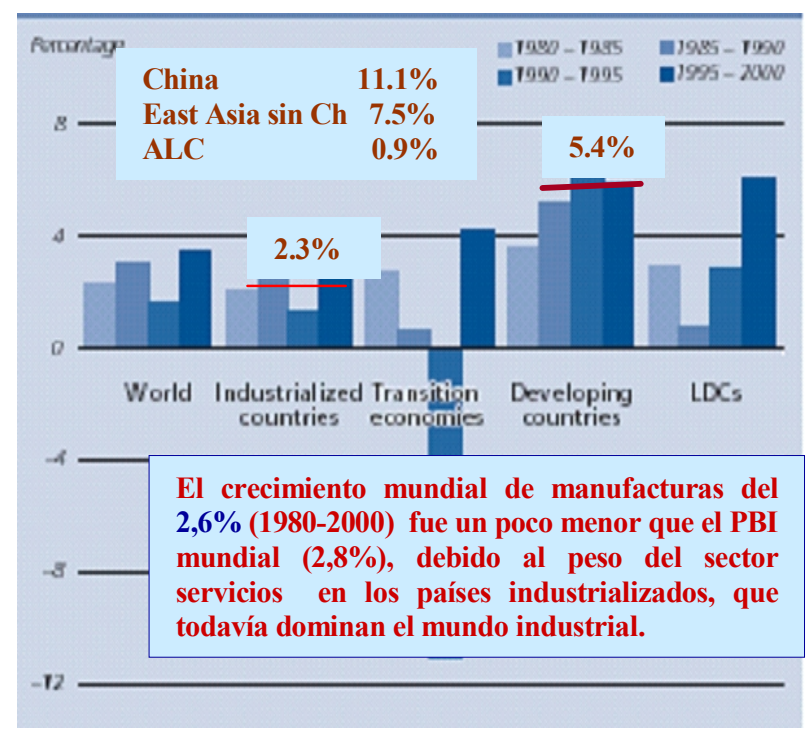

Figura 5. Crecimiento anual del MVA, 1980-2000 Fuente: ONUDI, 2004 ción en el ránking. En el Asia oriental: Salvo India, los países emergentes muestran progresivos MEJORES desempeños, incluyendo a China; ...pero en ALC: Salvo México, los demás países han perdido posiciones en la clasificación industrial, incluyendo al Perú.

\section{TENDENCIAS INDUSTRIALES 1980 AL 2000}

Dentro de esta amplia tendencia de 20 años, el desempeño de las regiones y los países ha exhibido considerables variaciones.

¿Cómo fue el crecimiento industrial? Debe resaltarse la diferencia de velocidad de crecimiento entre los dos bloques de países del mundo (ver Figura 5). En tanto que el mundo desarrollado crece al $2,3 \%$, el mundo en desarrollo lo hace a un ritmo superior al doble $(5,4 \%)$. Pero se presentan diferencias más notables al interior de la región de países en desarrollo; China y los países de Asia del Este crecieron a tasas por encima del $7 \%$ marcando el ritmo del desarrollo industrial. ALC presenta una pobre perfomance en su crecimiento industrial en dicho periodo: Sólo 0,9\%. Más aún, $\mathrm{ALC}$ en términos MVA per cápita ha disminuido en el periodo bajo análisis (ver Figura 6), cuando las economías de Asia del Este con y sin incluir a China muestran una tendencia creciente.

¿Cuál es el peso de las regiones en el MVA ?

Ver Figura 7. Lo notable de esta tendencia: aumento del mundo en desarrollo del $14 \%$ al $24 \%$ en el MVA, a costa del decrecimiento de los países desarrollados y de las economías en transición. Sin embargo, al interior del bloque en desarrollo como se aprecia en la Figura 8, Asia del Este ha duplicado su participación en el MVA del $29 \%$ al $54 \%$. Es la región más industrializada del mundo en desarrollo. Ha sido impulsor del reciente crecimiento industrial general. Pero en esta competencia en el mundo en desarro$\|$ lo,..... la región $A L C$ ha sido la principal perdedora, de estar en la cabeza en 1980 con una participación del $47 \%$, pasó en el 2000 a un precario segundo lugar con un $22 \%$.

¿Cuál es la contribución de la industria en el Producto mundial (GDP) ?

Ver Figura 9. En términos globales la participación del MVA mundial en el producto total en el periodo 1980-2000 prácticamente se ha mantenido en el promedio del $22 \%$. Los países desarrollados diminuyen su aporte y el mundo en desarrollo asume una mayor contribución. En este punto cabe indicar su alta contribución de las economías en transición deviene del 


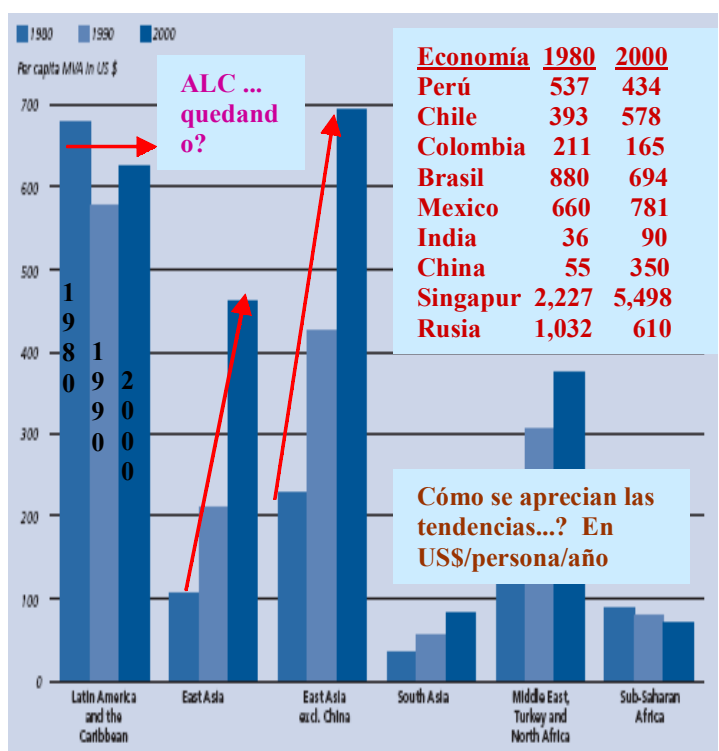

Figura 6 . Economías en Desarrollo: Tendencias en el MVA per cápita, 1980-2000 Fuente: ONUDI, 2004

énfasis en la industria pesada con que contaban en una situación más temprana.

Movimiento tecnológico en manufactura La innovación ha producido un rápido cambio tecnológico en la estructura industrial y en las habilidades para el manejo de actividades. La globalización, el uso de mejores prácticas de manufactura, maquinaria de punta y mejores organizaciones son algunas de las fuerzas que impulsan el ascenso técnico. En el Cuadro 2 se aprecia la composición tecnológica en la industria mundial.

Cuadro 2. Estructura tecnológica del MVA

\begin{tabular}{|c|c|c|c|c|c|c|}
\hline \multicolumn{7}{|c|}{ COMPOSICION TECNOLOOGCA DE MNA, 1980-2000 } \\
\hline & \multicolumn{3}{|c|}{ Participación \%1980 } & \multicolumn{3}{|c|}{ Participación \% 2000} \\
\hline Economías & $\mathrm{RB}$ & LT & MFI & $\mathrm{RB}$ & LT & $\mathrm{MH}$ \\
\hline$\overline{M u n d o}$ & 26.6 & 17.9 & 55.4 & 24.8 & 14.8 & 60 \\
\hline Industiales & 25.3 & 17.1 & 57.6 & 23.4 & 14.5 & 62 \\
\hline En transición & 27.3 & 26.0 & 46.6 & 34.3 & 23.5 & 42 \\
\hline En desarrollo & 36.5 & 227 & 40.8 & 31.1 & $16-0$ & 52 \\
\hline
\end{tabular}

Fuente: UNDO

RB : manufacturas basada en materias primas

LT : manufacturas basadas en actividades de baja a meda tecnología MHT : manufacturasbasadas en actividades de alta tecnología

$\checkmark$ A nivel mundial: Actividades MHT ganan en participación MVA a expensas de RBy LT

$\checkmark$ Países industriales siguten concentrando esfuerzos en actividades MHT y un minimo $\mathrm{en}$ actividades $\mathrm{RB}$ y $\mathrm{LT}$

$\checkmark$ Mundo en desarrollo arranca con ALTO \% de RB y BAJO MHT y finalizan con MAS ALTO MHT y MAS BAJORB que economíns en transición

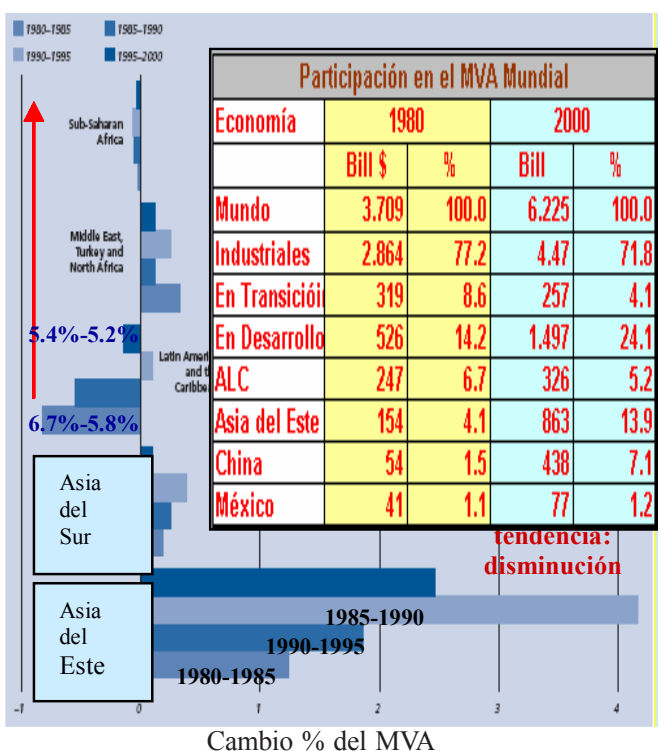

Figura 7 . Cambios en la Participación en el MVA Global, 1980-2000

Fuente: ONUDI, 2004

Los datos muestran un cambio hacia la producción de productos con mayor contenido tecnológico: producción MHT aumenta su participación en el MVA a expensas de RB y LT. Los países industrializados concentran mayores actividades en MHT que en RB y LT. En este caso la región en desarrollo ha mejorado su participación en actividades MHT, bajando en $\mathrm{RB}$, pero también en LT.; situación casi contraria ha sucedido en el caso de las economías en transición. En la Figura 10 se aprecia lo que sucede en la escena mundial: Asia del Este sube en todas las categorías tecnológicas, pero ALC se deteriora...

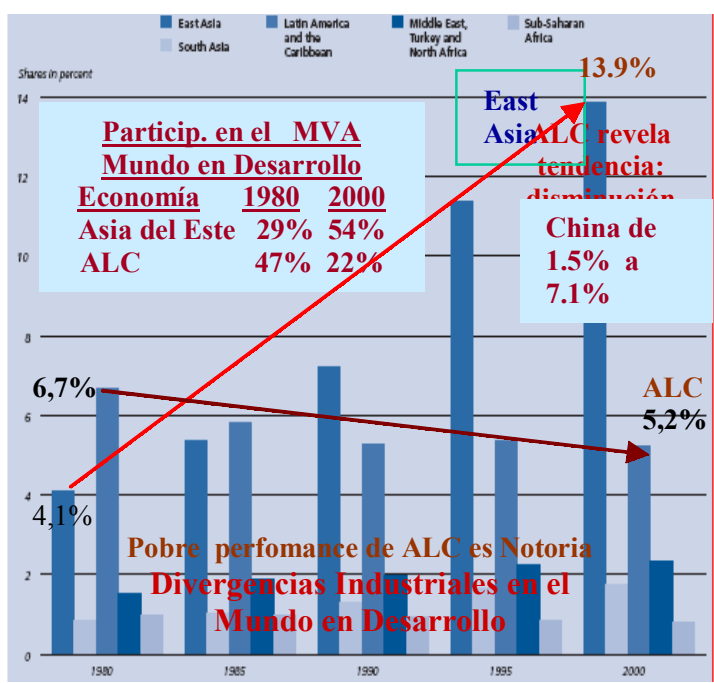

Figura 8. Participación en el MVA Mundial: 1980-2000 Fuente: ONUDI, 2004 
Cuadro 3 . Escenario Mundo en Desarrollo

\begin{tabular}{l|l|r|r}
\hline \multicolumn{4}{c}{ Participación en el MVA (\%) } \\
\hline Región/pais & Tecnología & $\mathbf{1 9 8 0}$ & $\mathbf{2 0 0 0}$ \\
\hline Asia del Este & RB & 31.4 & 25.8 \\
\hline & LT & 26.7 & 16.3 \\
\hline & MHT & 41.9 & 58.0 \\
\hline China & RB & 27.3 & 29.3 \\
\hline & LT & 35.3 & 14.5 \\
\hline & MHT & 47.4 & 56.1 \\
\hline Asia del Sur & RB & 25.0 & 26.5 \\
\hline & LT & 26.6 & 19.2 \\
\hline & MHT & 48.4 & 54.3 \\
\hline ALC & RB & 38.7 & 40.7 \\
\hline & LT & 19.9 & 11.9 \\
\hline & MHT & 41.5 & 47.4 \\
\hline México & RB & 40.4 & 47.5 \\
\hline & LT & 22.1 & 18.3 \\
\hline & MHT & 37.6 & 34.2 \\
\hline & Fuen
\end{tabular}

Fuente: UNIDO Scoreboard Database

La ONUDI señala además: En el Reporte 2002 de la US Nacional Science Board para una muestra de 68 países que contabilizan el $95 \%$ de la actividad industrial en el mundo, el ranking de 13 mayores países en la producción de productos de alta tecnología (HT) indica: En la mayoría de los países industrializados la manufactura total creció $2,7 \%$ y HT a $5,5 \%$; en los países en desarrollo fue de 4,8\% y 9,3\% respectivamente. La perfomance del sector HT es excepcionalmente fuerte en economías con mayor crecimiento, como es el caso de los países del este asiático.

Se registra varios países de ALC donde la producción HT está retrasada en la producción de manufactura: Chile, Colombia, Ecuador, Honduras, Panamá,

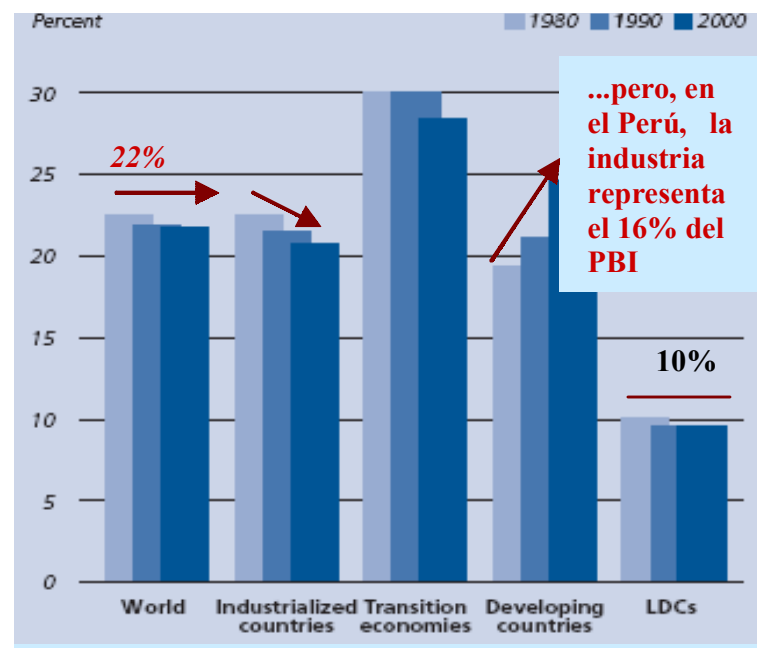

La contribución del MVA al PBI declina lentamente a nivel mundial, cediendo al sector SERVICIOS en los países ricos

Figura 9. Participación del MVA en el GDP, 1980-2000 Fuente: ONUDI, 2004
Cuadro 4. Exportaciones de Manufactura 1980-2000 (billones de dólares corrientes)

\begin{tabular}{|l|r|r|r|r|}
\hline & 1980 & 2000 & $\begin{array}{l}\text { (a) \% } \\
\text { Anual }\end{array}$ & $\begin{array}{l}\text { (b) } \% \\
\text { Anual }\end{array}$ \\
\hline Mundo & 1,234 & 4,925 & 7,5 & 2,6 \\
\hline Países industrializados & 1,010 & 3,407 & 6,6 & 2,3 \\
\hline Economías en transición & 62 & 196 & 6,3 & $(1,1)$ \\
\hline Países en desarrollo: & 162 & 1,322 & 11,8 & 5,4 \\
\hline LAC & 40 & 251 & 10,2 & 1,4 \\
\hline Asia del Sur & 7 & 54 & 11,0 & 0,9 \\
\hline Asia del Este & 84 & 908 & 13,4 & 9,0 \\
\hline China & 12 & 318 & 18,7 & 11,1 \\
\hline
\end{tabular}

(a) Tasa de crecimiento promedio anual de las exportaciones de manufactura (b) Tasa de crecimiento promedio anual del MVA Fuente: UNIDO Scoreboard Database

Perú, Uruguay y Venezuela, lo que explica la situación actual: ALC está mayormente especializado en actividades RB. Resalta además que industrias HT están más orientadas a la exportación que la manufactura como un todo, si bien hay excepciones, como es el caso de algunos países en ALC: Argentina, Brasil y Chile.

¿Cómo es el patrón tecnológico dentro de los países en desarrollo?

Si bien el bloque ha mejorado su estructura tecnológica, hay variadas diferencias en cada región y el patrón de crecimiento está muy influenciado por las economías de Asia del Este, como se desprende del Cuadro 3. Si bien, dicha región y ALC presentan la misma proporción en la actividad MHT en 1980, ALC se queda en el 2000. En Asia del Sur, India es el país dominante que pone la pauta del cambio.China sí impuso un cambio en el contenido tecnológico (MHT) en relación a México, que registró una pobre perfomance en este rubro.

\section{Rendimiento de las exportaciones}

Como se nota en el Cuadro 4, las exportaciones de manufactura han crecido más rápido que el MVA. En tanto que el crecimiento de las exportaciones en los países industrializados de 1980 al 2000 fue de 3,4 a 1, en los países en desarrollo fue: 9 a 1 . Al interior de éste último bloque se presentan diferencias importantes; ALC crece de 6 a 1, Asia del Sur: 8 a 1 y Asia del Este sorprende: 12 a 1, lo que es consistente con la velocidad de crecimiento de su industria.

Las economías en desarrollo consiguieron participación mundial básicamente a expensas de las economías industriales, doblaron exportaciones de manufactura de un 13\% a $27 \%$ (Ver Figura 9). Todavía los países industriales contabilizan los dos tercios del mercado mundial de exportaciones de manufactura. 


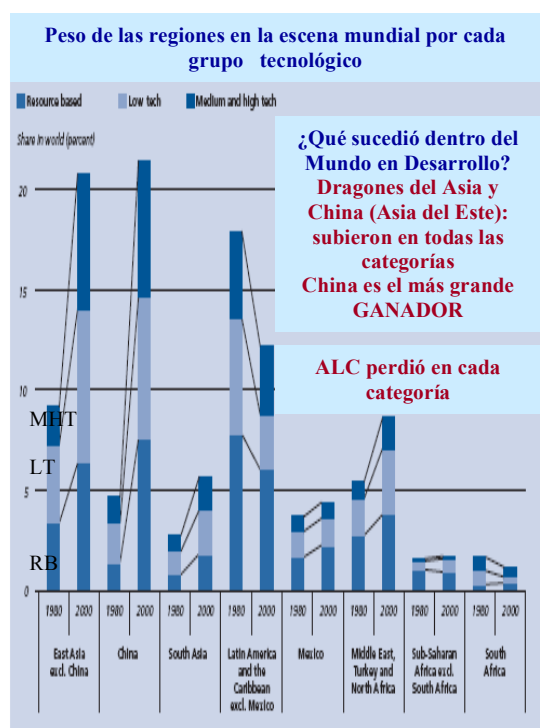

Figura 10. EscenaMundial:movimiento tecnológico, 1980y2000 Fuente: ONUDI, 2004

La tendencia del contenido tecnológico Ver Figura 11. Los productos de tecnología media (MT) que corresponden a la "industria pesada" son los que dominan la exportación mundial de manufacturas; sin embargo su participación se está reduciendo estacionariamente, de forma similar que los productos RB. Productos MT y HT representan el grueso de la actividad industrial de países maduros, pero su participación cae progresivamente. Los ganadores son los productos HT que comenzaron con $14 \%$ de participación en exportación de manufacturas y terminaron con $28 \%$ en el periodo bajo análisis.

La información también explica que los productos HT han tenido el más rápido crecimiento en los dos grandes bloques de países. Las economías desarrolladas disminuyen paulatinamente su participación en expor-

Cuadro 5. Participación de las Economías en Desarrollo en Exportaciones

\begin{tabular}{|l|r|r|r|}
\hline \multicolumn{4}{|c|}{$\begin{array}{c}\text { Países en Desarrollo: Participación de } \\
\text { las Exportaciones en el Mercado } \\
\text { Mundial }\end{array}$} \\
\hline En \% & 1981 & $\mathbf{2 0 0 0}$ & Aumento \% \\
\hline TOTAL & 13,0 & 27,0 & 14,0 \\
\hline RB & 22,5 & 25,5 & 3,0 \\
\hline LT & 24,5 & 39,0 & 14,5 \\
\hline MT & 6,0 & 17,5 & 11,5 \\
\hline HT & 9,5 & 32,5 & 23,0 \\
\hline
\end{tabular}

Fuente: ONUDI, Scoreboard Database.

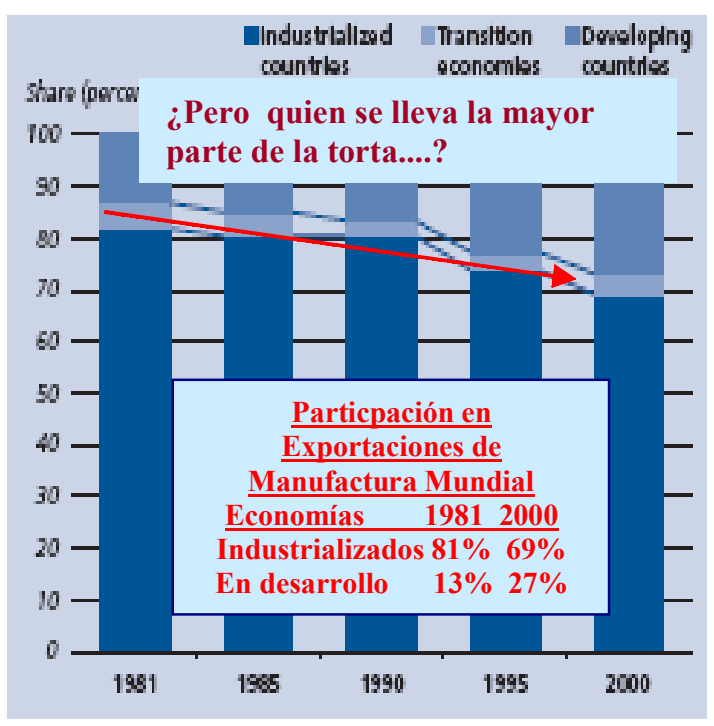

Figura 11. Participaciónenlaexportaciónmundial demanufacturas Fuente: ONUDI, 2004

taciones de productos RB y LT. Lo anterior es consistente con una estrategia o perspectiva de posicionamiento de mercado para tomar ventaja en productos más dinámicos, dado que exportar manufacturas RB o LT es menos atractivo que productos MT y HT. En el Cuadro 5 se visualiza con claridad la participación de las economías en desarrollo en cada categoría y los cambios registrados en el periodo 1981Participación de la Economías en Desarrollo-2000. En términos de valor, los países en desarrollo contabilizan un tercio de las exportaciones mundiales de productos $\mathrm{HT}$, equivalente a 113 billones de dólares.

Mundo en desarrollo: disparidades regionales en el desempeño de exportaciones Ver Figura 12. El desempeño de las exportaciones es muy desigual en el mundo en desarrollo, más aún

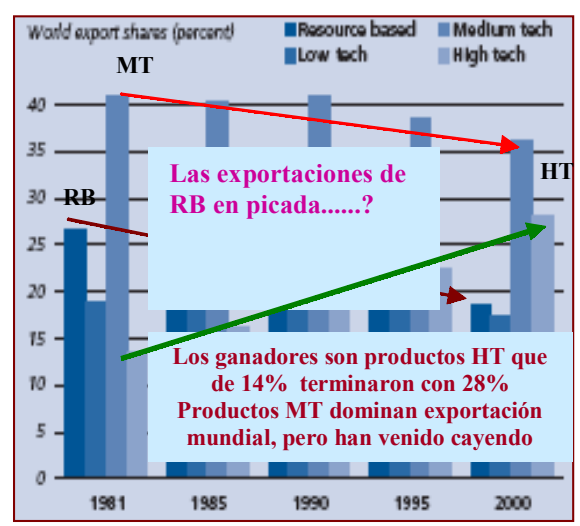

Figura 12. Estructuratecnológica:Exportaciónmundialdemanufacturas Fuente: ONUDI, 2004 


\section{Cuadro 6. Matriz de posicionamiento}

Para analizar el modelo de competencia de una región, se debe

VISUALIZAR la distribución de exportaciones en una MATRIZ relacionando el dinamismo de sus exportaciones al del mercado mundial

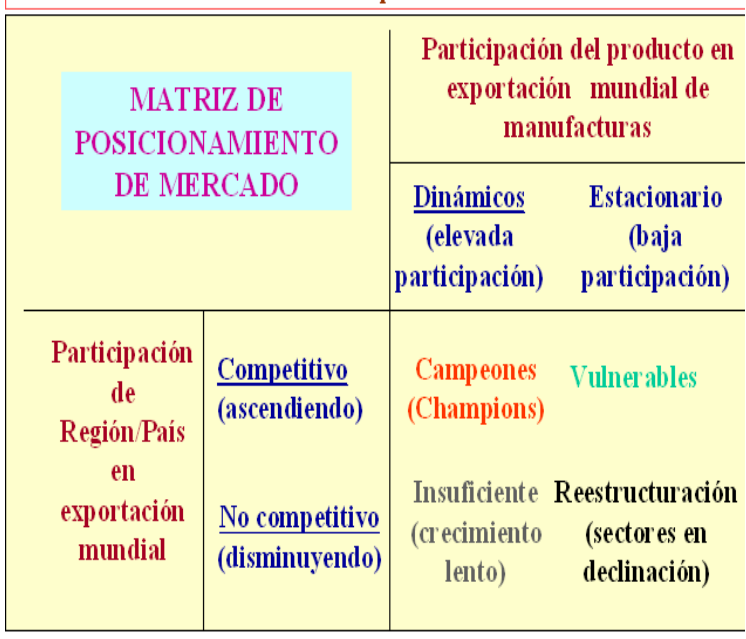

de lo ocurrido con el VAM. Asia del Este con China incluida, le corresponde casi el $70 \%$ de las exportaciones de manufacturas del mundo en desarrollo en el 2000 , frente a un $52 \%$ en 1981; su participación es mucho mayor en productos $\mathrm{HT}$, pasó de $73 \%$ a $85 \%$, y más bajo en productos RB, pasando de $39 \%$ a $46 \%$. Aquí el desempeño de ALC, salvo México también fue decepcionante, a pesar que adoptó una política liberal más rápidamente que las otras regiones en desarrollo.

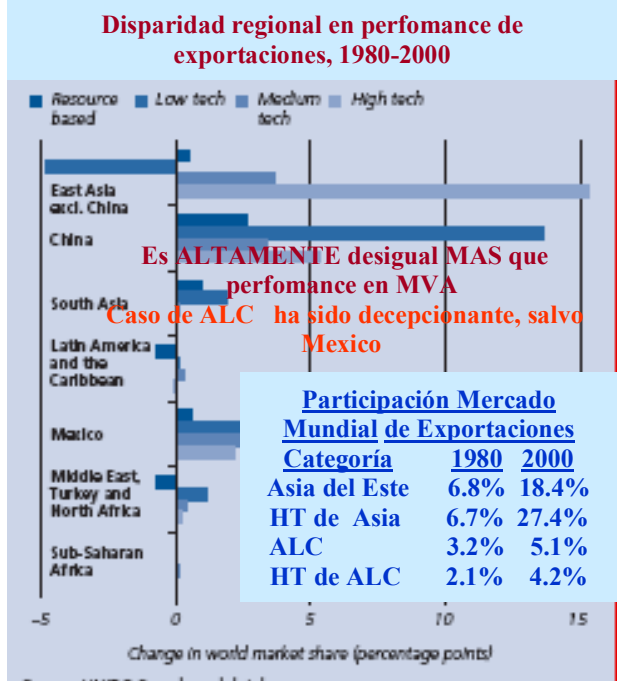

Figura 13. Economías en desarrollo: Cambios en la participación de mercado Fuente: ONUDI, 2004

\section{ANALI Z AR COMPETITIVIDAD INDUSTRIAL}

La ONUDI (8) usa una matriz de posicionamiento en el mercado mundial como el que se muestra en el Cuadro 6. Se presentan cuatro cuadrantes:

1. "Champion (Campeón)": La mejor posición competitiva, con productos dinámicos en la cual la región gana participación en el mercado.

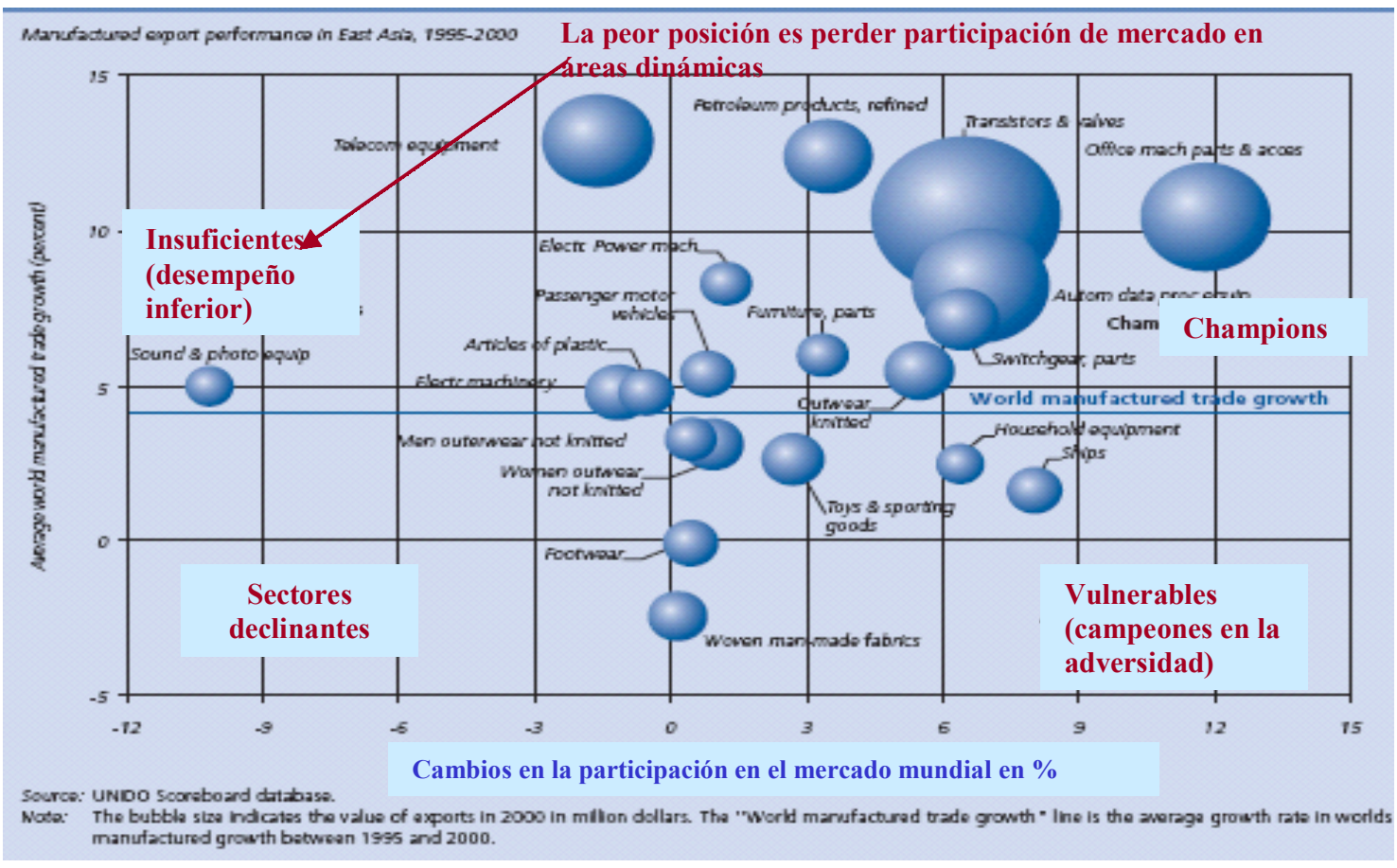

Figura 14. East Asia: Posicionamiento de Mercado en 20 Productos líderes de exportación 


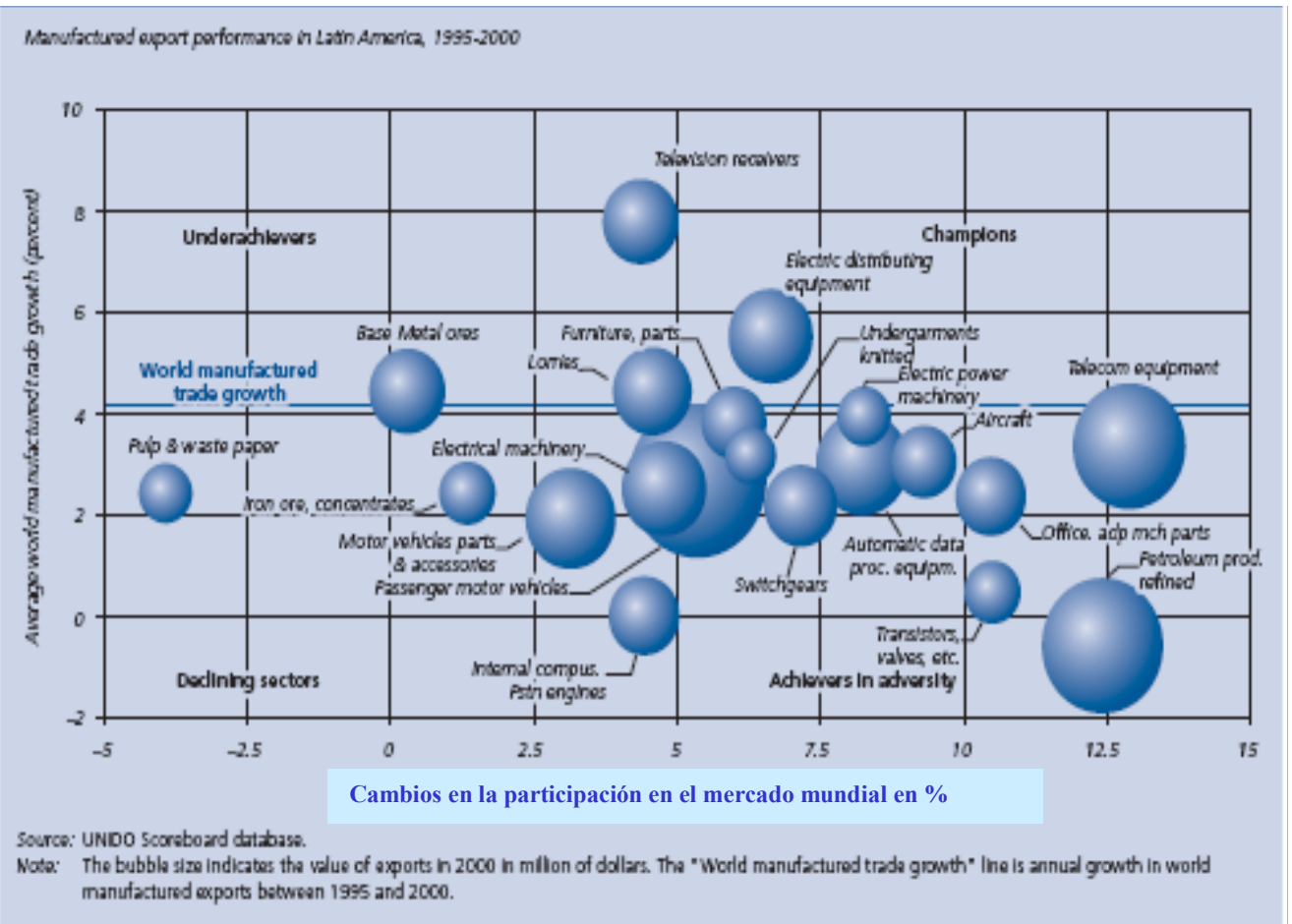

Figura 15. ALC: Posicionamiento de Mercado en 20 Productos líderes de exportación

2. "Underachievers (Desempeño inferior)", también denominados INSUFICIENTES (oportunidades perdidas): La peor posición competitiva y corresponde a pérdida de participación de mercado en áreas dinámicas.

3. "Achievers in adversity (Campeones en adversidad)". VULNERABLES: Productos estacionarios en el mercado mundial, en el cual la región gana mercado.

4. "Declining sectors" (Declive)": Productos estacionarios en el mercado mundial, en el cual la región pierde participación.

Se debe evaluar la densidad de cada cuadrante así como su estructura tecnológica. Cabe anotar que en la matriz, "World manufactured trade grown" como la línea de crecimiento promedio mundial $(4,1 \%)$ en exportación de manufacturas entre 1995 y 2000 . Sólo con fines ilustrativos se presenta esta matriz para la región Asia del Este y ALC en las Figuras 13 y 14 respectivamente. Asia del Este registra una población mayor de productos complejos que ALC, debiendo anotarse que el perfil de ALC está influenciado por la perfomance de México. Estos diagramas sugieren entonces, que Asia del Este y México son los mejores posicionados para futuros crecimientos de exportaciones y ascenso en categorías tecnológicas superiores.

En la Figura 15 (1) se grafica la posición competitiva de algunos países de ALC, sugiriéndose el camino industrial deseado. La ONUDI (5) destaca que entre los 40 países peor clasificados en desempeño industrial entre 1995 y 1998 se encuentran: Perú, Colombia, Venezuela, Panamá, Ecuador, Nicaragua, Jamaica, Paraguay, entre otros países del mundo, situación que se evidencia en la referida Figura 16.

En este contexto se levantan en el Perú, voces de alerta y cambio. García (3) señala "La estrategia destinada a cimentar bien un primer piso de crecimiento económico es crear fábricas que valoren nuestras materias primas", para lo cual se requiere "ingeniería política". Oppenheimer (10) sugiere "El progreso pasa por la producción de bienes cada vez más sofisticados".

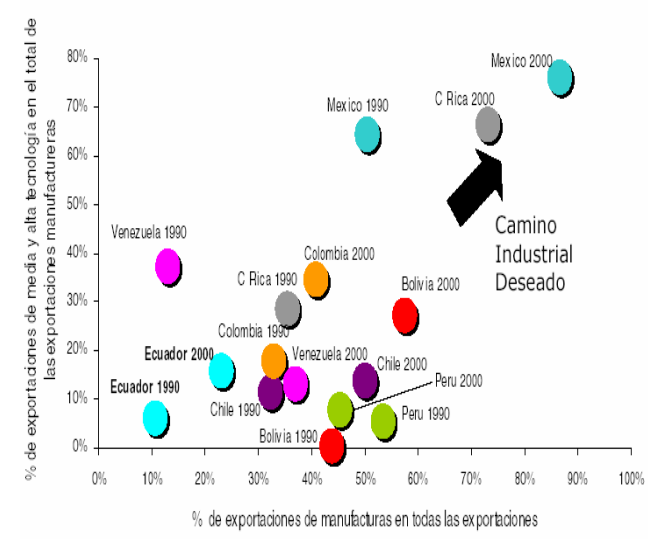

Figura 16. ALC: Competitividad Industrial Fuente: Albaladejo M., Consultor de ONUDI, 2004 


\section{CONCLUSIONES}

En general, en los últimos 20 años se ha observado un desplazamiento en la composición tecnológica de las manufacturas de actividades basadas en recursos y de baja tecnología a actividades de mediana y alta tecnología.

Los sectores de alta tecnología han crecido mucho más rápido que los sectores basados en recursos y de baja tecnología. Entre 1990 y 2000, el sector manufacturero global creció al $6.6 \%$, los productos HT al $11 \%$. Sectores con alto componente tecnológico (media y alta tecnología) son menos vulnerables a la competición exterior porque tienen una mayor barrera de entrada. Las actividades $\mathrm{MHT}$ son la mayor parte de manufacturas en la mayoría de países con sectores industriales maduros, lo que sugiere que el principal impulsor de MHT es contar con alta tecnología que tecnología media. Sectores con alto componente tecnológico ofrecen mayor perspectivas para el aprendizaje y la innovación.

Además hay factores estructurales que ejercen gran influencia en el desempeño industrial: capacidades, aptitudes (recursos humanos), esfuerzo tecnológico $(I+D)$, inversión extranjera directa del exterior (IED), transferencia de tecnologías e infraestructura moderna. Estos factores exhiben los mejores valores en los países industrializados, que marcan la vanguardia en I+D. Sin embargo, en este contexto en ALC se revela una tendencia: Disminución de la participación en el MVA mundial, aspecto que es necesario revertir.

En resumen, la industria constituye el catalizador del crecimiento para los países en desarrollo, por ser el exponente del desarrollo tecnológico y la innovación, y por su 'efecto de empuje' en otros sectores de la economía.

\section{RECOMENDACIONES}

Los resultados anteriores abonan la propuesta planteada por Porlles, J. y otros autores en un trabajo anterior registrada en esta Revista (12), propiciando un cambio radical en el modelo productivo nacional, movimiento que se hace más intenso en el Perú. Un impulso decisivo, transformador del modelo actual, implica en esta etapa el aprovechamiento de las ventajas comparativas del Perú, en los grandes ejes industriales siguientes:

- Química de los metales a partir de los metales que se exportan actualmente.

- Petroquímica a partir del uso del Gas de Camisea.

- Agroindustria exportadora.

- Industria textil.

- Robótica y automatización industrial.
Se sugiere además que los responsables del gobierno peruano prioricen la adopción de acciones para el fortalecimiento de la capacidad competitiva de la industria nacional: promoviendo e incentivando la productividad y capacidad industrial en actividades manufactureras de mayor valor agregado y contenido tecnológico en la perspectiva de las tendencias industriales actuales y futuras.

\section{REFERENCIAS BIBLIOGRÁFICAS}

1. Albaladejo, M. (2004). La Experiencia de la ONUDI en el Apoyo a las Políticas de Competitividad Industrial. Consultor de la ONUDI. Exposición en Bogotá, 11 de Mayo 2004.

2. Banco Mundial (2005). Informe sobre el DesarroIlo Mundial 2005: Un mejor clima para la inversión en beneficio de todos. Septiembre 2004.

3. García, G., (2005). Comentario: Verdadero primer piso. Diario La Republica-Perú, publicado el 19.09.05.

4. ONUDI (2003). Organización de las Naciones Unidas para el Desarrollo Industrial. Informe sobre el Desarrollo Industrial 2002-2003.

5. ONUDI (2003), op. cit., pp.

6. ONUDI (2004). Organización de las Naciones Unidas para el Desarrollo Industrial. Informe sobre el Desarrollo Industrial 2004. Julio 2004.

7. ONUDI (2004), op. cit., pp. 12 .

8. ONUDI (2004), op. cit., pp. 154.

9. ONUDI (2005). Organización de las Naciones Unidas para el Desarrollo Industrial. Informe sobre el Desarrollo Industrial 2005: Capability building for catching-up. Noviembre 2005.

10.Oppenheimer, A., (2005). Comentario Internacional: La maldición de los recursos naturales. Diario El Comercio-Perú, publicado el 13.09.05.

11.Porlles, J., y otros (2005). Posición Competitiva y Modelo Productivo en el Perú. Revista Industrial Data, Facultad de Ingeniería Industrial, UNMSM. Volumen 8, Nro 2, Julio-Diciembre 2005.

12.Porlles, J., op.cit., pp. 49-50.

13. Programa de las Naciones Unidas para el Desarrollo (PNUD),(2005). El Informe del Desarrollo Humano. Lima, Perú. 